\title{
Diabetes y su impacto en el territorio periodontal
}

\author{
Diabetes and its impact in periodontal tissues
}

\author{
Smith $\mathrm{P}^{1}$, Retamal $\mathrm{I}^{1}$, Cáceres $\mathrm{M}^{1}$, Romero $\mathrm{A}^{1}$, Silva $\mathrm{D}^{1}$, Arancibia $\mathrm{R}^{1}$, Martínez $\mathrm{C}^{1}$
}

\section{RESUMEN}

Diabetes y enfermedad periodontal corresponden probablemente al mejor ejemplo de cómo una enfermedad sistémica puede tener un efecto en el territorio periodontal. Si bien esta asociación ha sido extensamente estudiada, muchas de las asociaciones propuestas presentan contradicciones. En la presente revisión de la literatura se analizan los siguientes tópicos relevantes para la práctica clínica en periodoncia e implantología: i) Identificación de enfermedad periodontal severa y su capacidad para diagnosticar casos de diabetes; ii) Efectos de la diabetes sobre la enfermedad periodontal; iii) Efectos de la diabetes sobre la reparación periodontal y periimplantaria; iv) Efecto del tratamiento periodontal sobre el control metabólico de la diabetes.

Rev. Clin. Periodoncia Implantol. Rehabil. Oral Vol. 5(2); 90-92, 2012.

Palabras clave: Diabetes, enfermedad periodontal, tejidos periodontales, terapia periodontal.

\begin{abstract}
Diabetes and periodontal disease correspond to conditions that probably exemplify how a systemic disease may have a strong impact in the periodontium. Although this association has been studied for several years, many of these studies still show contradictory results. The present review analyses the following questions relevant for the clinician in the fields of periodontology: i) Value of the diagnosis of severe periodontitis and its capacity to identify previously un-diagnosed cases of diabetes; ii) Effects of diabetes on periodontal disease; iii) Effects of diabetes on periodontal and periimplant tissue repair and regeneration and; iv) Effect of periodontal therapy on the metabolic control of diabetes.
\end{abstract}

Rev. Clin. Periodoncia Implantol. Rehabil. Oral Vol. 5(2); 90-92, 2012.

Key words: Diabetes, periodontal disease, periodontal tissues, periodontal therapy.

\section{INTRODUCCIÓN}

Diabetes y enfermedad periodontal corresponden a dos condiciones estudiadas probablemente desde los inicios de la llamada medicina periodontal. Si bien se ha avanzado en esclarecer el vínculo entre éstas, los resultados de muchos estudios son aun contradictorios. En la presente revisión se entrega una visión general sobre los mecanismos patogénicos y características clínicas de la diabetes y se indaga sobre cuatro preguntas de alta relevancia para la práctica clínica en periodoncia e implantología: ¿Cuál es el efecto de la diabetes sobre la evolución de la enfermedad periodontal? ¿De qué manera la identificación de un paciente con elevado daño periodontal puede ser empleado como un indicador de diabetes? ¿Cuáles son los efectos de la diabetes sobre la regeneración periodontal y neoformación de tejidos en implantología? ¿Cuál es el impacto de la terapia periodontal sobre la evolución y control metabólico de la diabetes?

\section{GENERALIDADES DE DIABETES}

La diabetes corresponde a un grupo de enfermedades metabólicas caracterizadas por la mantención de una condición de hiperglicemia que puede tener su origen en defectos en la secreción de insulina, en alteraciones de la acción de la insulina sobre las células blanco $o$ en ambos procesos ${ }^{(1,2)}$. La hiperglicemia crónica que ocurre en la diabetes está asociada a daño crónico y disfunción de diversos órganos y tejidos tales como ojos, riñones, nervios, corazón, vasos sanguíneos y dentición ${ }^{(1,2)}$. Diferentes procesos patológicos han sido involucrados en el desarrollo de diabetes. Estos incluyen la destrucción de las células beta del páncreas con la consecuente deficiencia en la producción de insulina, así como defectos en la capacidad de células musculares y hepáticas para responder a esta hormona. Tanto el defecto en la producción de insulina como la incapacidad de las células para responder a esta suelen coexistir en un mismo paciente y es complejo el identificar cual es la fuente primaria que genera la hiperglicemia(3). Las complicaciones más frecuentemente vinculadas a la evolución prolongada de la diabetes incluyen el desarrollo de retinopatías con potencial pérdida de la visión, nefropatías que pueden llevar a la falla renal, úlceras de extremidades y neuropatía periférica que puede derivar en síntomas gastrointestinales, genitourinarios, cardiovasculares y disfunción sexual ${ }^{(3)}$. Los pacientes con diabetes tienen además un mayor riesgo de aterosclerosis ${ }^{(2,3)}$. La gran mayoría de los individuos con diabetes caen en dos grandes categorías de esta enfermedad. El primer grupo corresponde a diabetes tipo 1 ( $5 \%$ aproximado del total de casos de esta enfermedad) y la causa esencial es la deficiencia en la secreción de insulina. El segundo grupo (95\% aproximado del total) corresponde a diabetes tipo 2 y presenta una combinación de resistencia a insulina y una inadecuada secreción compensatoria de esta última hormona ${ }^{(2,3)}$.

\section{DIAGNÓSTICO DE ENFERMEDAD PERIODONTAL SEVERAY SU CAPACIDAD PARA IDENTIFICAR CASOS DE DIABETES NO DIAGNOSTICADA PREVIAMENTE}

La diabetes tipo 2 es una entidad patológica difícil de identificar y se ha estimado que cerca de un cuarto de los pacientes diabéticos en EE.UU. no han sido diagnosticados ${ }^{(4)}$. Existen evidencias que muestran además que el diagnóstico temprano de la diabetes puede mitigar sus complicaciones y mejorar el estado de salud de sus afectados ${ }^{(5)}$. Por otro lado, la pre-existencia de periodontitis puede predecir una peor evolución de enfermedades cardiovasculares y renales en pacientes diabéticos ${ }^{(6,7)}$. Estos antecedentes ponen en relieve la importancia del diagnóstico precoz de esta enfermedad metabólica. Un estudio reciente muestra que la determinación de la presencia de sacos periodontales profundos y de pérdida de 4 o más dientes permite identificar cerca de un $73 \%$ de casos de pacientes diabéticos. Al agregar a este examen la identificación de una hemoglobina glicosilada $(\mathrm{HbA} 1 \mathrm{c}) \geq 5.7 \%$, esta capacidad de predicción sube a un $92 \%$. Esta evidencia permite proponer que el examen periodontal con hallazgos de EP avanzada podría ser utilizado como un elemento importante para sospechar la existencia de diabetes ${ }^{(8,9)}$.

1. Carrera de Odontología, Facultad de Medicina. Pontificia Universidad Católica de Chile. Chile.

Correspondencia autor: Patricio Smith. psmithf@uc.cl. Laboratorio de Fisiología Periodontal, Carrera de Odontología. Facultad de Medicina, Pontificia Universidad Católica de Chile. Chile. Trabajo recibido el 02/08/2011. Aprobado para su publicación el 01/11/2011. 
EFECTOS DE DIABETES SOBRE LA ENFERMEDAD PERIODONTAL

Diferentes estudios han evaluado el potencial impacto de la diabetes en el tejido periodontal. La mayor parte de ellos muestra que la hiperglicemia crónica puede alterar de manera significativa la salud de este territorio comprometiendo la fisiología de este tejido a distintos niveles. La pérdida de inserción periodontal parece estar estrechamente vinculada al control metabólico de la diabetes. Es así como la presencia de un pobre control de estas enfermedad, medida a través de los niveles plasmáticos de hemoglobina glicosilada (HbA1c), se asoció con mayor prevalencia, severidad y extensión de la enfermedad periodontal ${ }^{(10-12)}$. Se ha visto además que tanto los individuos diabéticos no insulinodependientes como los insulino-dependientes presentan un riesgo incrementado para el desarrollo de enfermedad periodontal(10). Estos antecedentes han permitido proponer que el mal control metabólico de la diabetes es un factor importante que puede modular negativamente la evolución de la enfermedad periodontal. Un estudio pionero en este sentido fue el realizado por el grupo de Grossi y Genco durante los años 90' en la población de indios Pima en EE.UU. Estos trabajos mostraron que la diabetes puede actuar como un importante factor de riesgo para la pérdida de inserción periodontal con un odds ratio de $2.32^{(13)}$. Esta asociación parece ser extremadamente estrecha en la población de indios Pima antes mencionada, en la cual los sujetos diabéticos noinsulino dependientes presentaron una pérdida ósea 2.6 veces mayor en comparación con pacientes no diabéticos ${ }^{(13)}$. Por otro lado, si las condiciones de mantención y cuidados periodontales son adecuadas a lo largo del tiempo, la evolución de pacientes diabéticos puede ser similar tanto en insulino como en no insulino dependientes ${ }^{(14)}$. Este hecho permite resaltar que la hiperglicemia crónica podría explicar los efectos periodontales de la diabetes.

\section{EFECTOS DE LA DIABETES SOBRE LA REPARACIÓN Y REGENERACIÓN PERIODONTAL Y PERI-IMPLANTARIA}

Actualmente la diabetes es considerada una contraindicación relativa para la instalación de implantes óseointegrados, decisión que depende básicamente del control de la glicemia(15). La reparación periodontal en pacientes diabéticos, particularmente en condiciones de pobre control metabólico de la enfermedad, también manifiesta importantes deficiencias ${ }^{(16,17)}$. En consistencia con estas asociaciones, la hiperglicemia puede generar defectos en la neoformación ósea y la integración de implantes, observándose en modelos animales una respuesta hasta un $30 \%$ menor en la formación de tejido óseo alrededor de implantes al ser comparados con animales controles ${ }^{(18-20)}$. Por otro lado, al analizar los resultados de la instalación de implantes en humanos, los resultados muestran variaciones de entre un 0 y $15 \%$ de menor formación ósea en presencia de diabetes ${ }^{(21-27)}$. Esta mayor divergencia de los resultados podría derivar de la amplia variabilidad en la evolución y control clínico de la diabetes en humanos. La conclusión general de estos estudios es que defectos en el control de la glicemia podrían explicar esta respuesta deficitaria en la reparación periodontal y periimplantaria en pacientes diabéticos ${ }^{(28)}$.

La diabetes es una enfermedad que conocidamente compromete la respuesta inflamatoria y reparativa del organismo. Es posible considerar además que los tejidos periodontales, al estar expuesta a un constante desafío infeccioso y traumático podrían ser particularmente sensibles al efecto de la diabetes. La hiperglicemia podría actuar como mecanismo efector común de las alteraciones generadas por la diabetes en los tejidos periodontales. En este sentido, se ha observado que niveles elevados de glicemia pueden inhibir la proliferación celular en osteoblastos y la producción de colágeno durante las etapas tempranas de la formación del callo óseo, lo que resulta en una menor neoformación ósea y una reducida capacidad mecánica del hueso regenerado(29-33).

Por otro lado, la modulación de la respuesta inflamatoria en diabéticos podría ser importante en la generación de las complicaciones descritas e nivel periodontal. Utilizando monocitos derivados de diabéticos tipo 1, Salvi y col. observaron que estas células respondían a Lipopolisacáridos (LPS) de Porphyromonas gingivalis liberando niveles aumentados de TNF- $\alpha$ en comparación con pacientes no diabéticos pareados por su estado periodontal(34). En otro estudio de los mismos autores, los diabéticos tipo 1 mostraron niveles incrementados de
PGE2 e IL-1 $\beta$ en el fluido crevicular en comparación con controles no diabéticos pareados por severidad de enfermedad periodontal. Más aun, monocitos de estos pacientes estimulados con LPS de Escherichia coli y $P g$ liberaron niveles significativamente superiores de PGE2 e IL-1 $\beta$ en comparación con monocitos de pacientes no diabéticos con enfermedad periodontal(35). Estos estudios sugieren que los pacientes diabéticos pueden tener una respuesta inflamatoria más agresiva que pacientes no diabéticos a niveles comparables de enfermedad periodontal. En relación a la reparación periodontal, TGF- $\beta 1$ es un factor de crecimiento que promueve la producción de colágeno tipo I y la diferenciación de miofibroblastos, facilitando así la regeneración de la matriz extracelular( ${ }^{(36)}$. Se ha observado que tanto TNF- $\alpha$ como IL-1 $\beta$ pueden inhibir la producción de colágeno tipo I y la diferenciación de miofibroblastos en cultivos primarios de células mesenquimales de piel ${ }^{(37)}$. Es posible entonces que la mantención de la respuesta inflamatoria en pacientes diabéticos pueda retardar la reparación y regeneración de tejidos a nivel periodontal.

La mantención de niveles elevados de glicemia induce la glicosilación de proteínas con múltiples efectos sobre la remodelación tisular. En células en cultivo, la glicosilación de fibronectina puede alterar la capacidad de adhesión de fibroblastos gingivales y de ligamento periodontal. Otra respuesta fisiológica alterada al inducir la glicosilación de fibronectina es una reducción de la velocidad de migración celular, lo que tiene importantes repercusiones sobre la regeneración de los tejidos afectados ${ }^{(38)}$. Un efecto funcional importante derivado de la glicosilación del colágeno es la alteración en las células para adherirse a esta proteína y el defecto consecutivo en la degradación del colágeno por fagocitosis de esta macromolécula ${ }^{(39)}$. La diabetes puede promover la apoptosis de fibroblastos y osteoblastos, disminuyendo la masa crítica de células involucradas en la reparación de heridas ${ }^{(16)}$. Los principales efectos de la diabetes sobre la reparación periodontal se muestran en la Figura 1.

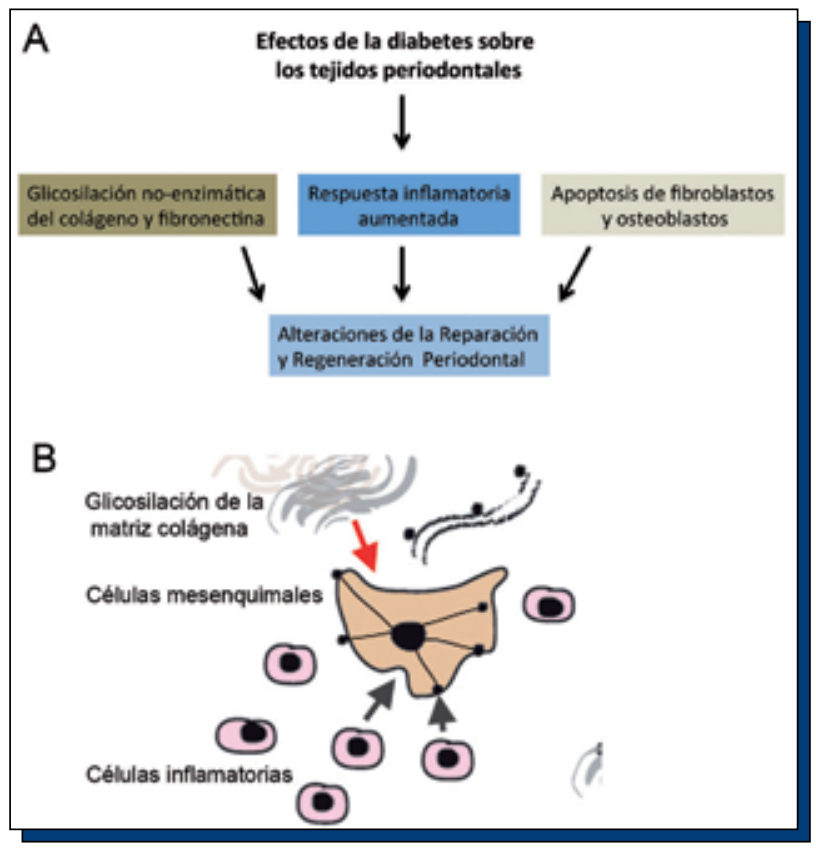

Figura 1. En el esquema se representa la potencial interacción entre células inflamatorias que en la diabetes pueden secretar mayores niveles de mediadores como TNF- $\alpha$. Está última citoquina puede alterar la funcionalidad de células mesenquimales disminuyendo, por ejemplo, su capacidad de remodelación de heridas (flecha gris). Por otro lado, la glicosilación de proteínas de la matriz puede alterar la interacción entre las células mesequimales y esta matriz colágena llevando a una respuesta reparativa aberrante.

\section{EFECTO DE LA TERAPIA PERIODONTAL SOBRE LA EVOLUCIÓN DE LA DIABETES}

Los primeros estudios en mostrar un efecto del tratamiento de la enfermedad periodontal sobre la diabetes fueron desarrollados por Grossi et al y mostraron que el tratamiento de la periodontitis era 
capaz de reducir los niveles de $\mathrm{HbA} 1 \mathrm{c}$ en la población de indios $\mathrm{Pima}^{(40)}$. Una serie de estudios han precedido a este trabajo pionero y un meta-análisis que reunió la información derivada de 10 estudios de intervención mostró una reducción de $0.38 \%$ en los niveles de $\mathrm{HbA} 1 \mathrm{c}$, de $0.66 \%$ al restringir el análisis a pacientes diabéticos tipo 2 y de $0.71 \%$ al considerar el uso de antibióticos durante el tratamiento. Sin embargo, estos valores de reducción en los niveles de hemoglobina glicosilada no alcanzaron niveles de significancia estadística(41). Es posible que estas diferencias en el control metabólico de la diabetes en respuesta al tratamiento de la enfermedad periodontal se deban a la heterogeneidad de las poblaciones que han sido estudiadas hasta el momento. Por otro lado, la población de indios Pima representa a un grupo étnicamente homogéneo y en donde es posible que las estrategias de tratamiento y de mantención periodontal utilizadas hayan permitido un mejor control del estado periodontal de estos pacientes ${ }^{(41-44)}$. La evidencia a la fecha permite pensar que el tratamiento de la enfermedad periodontal es capaz contribuir al control de la glicemia. Sin embargo, la magnitud y significancia clínica de este efecto aún requiere de nuevos y mejores estudios. en la Figura 2

Las principales conclusiones de este artículo son presentadas 1. The Expert Committee on the Diagnosis and Classification of Diabetes Mellitus:
Report of the Expert Committee on the Diagnosis and Classification of Diabetes Report of the Expert Committee on the Diagno

2. The Expert Committee on the Diagnosis and Classification of Diabetes Mellitus: Follow2. The Expert Committee on the Diagnosis and Classification of Diabetes Mellitus: Follow3. Position statement. American Diabetes Association. Diagnosis and Classification of Diabetes Mellitus. Diabetes Care, 2006; 29: S1: S43-S48.

4. Centers for Disease Control and Prevention-2011. National diabetes fact sheet: National estimates and general information on diabetes and prediabetes in the United States, 2010. Atlanta, GA: US Department of Health and Human Services, Centers for Disease Control and Prevention.

5. American Diabetes Association. Standards of medical care in diabetes-2010. Diabetes Care 33(Suppl 1):11-61; erratum in Diabetes Care, 2010; 33: 693.

6. Saremi A, Nelson RG, Tulloch-Reid M, Hanson RL, Sievers ML, Taylor GW. Periodontal disease and mortality in type 2 diabetes. Diabetes Care, 2005; 28: 27-32. 7. Shultis WA, Weil EJ, Looker HC, Curtis JM, Shlossman M, Genco RJ. Effect of periodontitis on overt nephropathy and end-stage renal disease in type 2 diabetes. Diabetes Care, 2007; 30: 306-311.

8. Lalla E, Kunzel C, Burkett S, Cheng B, Lamster IB. Identification of unrecognized diabetes and pre-diabetes in a dental setting. J Dent Res, 2011; 90: 855-860.

9. Li S, Williams PL, Douglass CW. Development of a clinical guideline to predict undiagnosed diabetes in dental patients. J Am Dent Assoc, 2011; 142: 28-37. 10. Tervonen T, Oliver RC. Long-term control of diabetes mellitus and periodontitis. J Clin Periodontol, 1993; 20: 431-435.

11. Loe H. Periodontal disease. The sixth complication of diabetes. Diabetes Care, 1993; 16: 329-334.

12. Moles DR. Evidence of an association between diabetes and severity of periodontal disease. Evid Based Dent, 2006; 7: 45

13. Grossi SG, Zambon JJ, Ho AW, Koch G, Dunford RG, Machtei EE, Norderyd OM, Genco RJ. Assesment of risk for periodontal disease. I. Risk indicators for attachment loss. J Periodontol, 1994; 65: 260-267.

14. Westfelt $E$, Rylander $\mathrm{H}$, Blohme G, Jonnason $\mathrm{P}$ Lindhe J. The effect of periodontal therapy in diabetics. Results alter 5 years. J Clin Periodontol, 1996; 23: 92-100. 15. Beikler T, Flemmig TF. Implants in the medically compromised patient. Crit Rev Oral Biol Med, 2003; 14: 305-316.

16. Desta T, Li J, Chino T, Graves DT. Altered fibroblast proliferation and apoptosis in diabetic gingival wounds. J Dent Res, 2010; 89: 609-614.

17. Santos VR, Lima JA, De Mendonça AC, Braz Maximo MB, Faveri M, Duarte PM. Effectiveness of full-mouth and partial-mouth scaling and root planing in treating chronic periodontitis in subjects with type 2 diabetes. J Periodontol, 2009; 80: 1237-1245. 18. Nevins ML, Karimbux NY, Weber HP, Giannobile WV, Fiorellini JP. Wound healing around endosseous implants in experimental diabetes. Int J Oral Maxillofac Implants, 1998; 13: 620-629

19. Gerritsen M, Lutterman JA, Jansen JA. Wound healing around boneanchored percutaneous devices in experimental diabetes mellitus. J Biomed Mater Res, 2000; 53: 702-709.

20. McCracken M, Lemons JE, Rahemtulla F, Prince CW, Feldman D. Bone response to titanium alloy implants placed in diabetic rats. Int $\mathrm{J}$ Oral Maxillofac Implants, 2000; 15: 345-351.

21. Shernoff AF, Colwell JA, Bingham SF. Implants for type 2 diabetic patients: Interim report. Implant Dent, 1994; 3: 183-189.

22. Garrett NR, Kapur KK, Hamada MO, Roumanas ED, Freymiller E, Han T et al. A randomized clinical trial comparing the efficacy of mandibular implant-supported overdentures and conventional dentures in diabetic patients: Part II. Comparisons of masticatory performance. J Prosthet Dent, 1998; 79: 632-640.

23. Fiorellini JP, Chen PK, Nevins M, Nevins ML. A retrospective study of dental implants in diabetic patients. Int J Periodontics Restorative Dent, 2000; 20: 366-373.

\section{CONCLUSIONES}

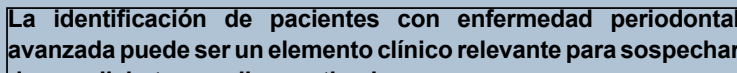
de una diabetes no diagnosticada.

Diferentes estudios muestran que la diabetes está asociada con un mayor riesgo de enfermedad periodontal y que puede modular negativamente la reparación periodontal y peri-implantaria.

El tratamiento de la enfermedad periodontal puede tener un efecto positivo en el control metabólico de la diabetes, medido a través de los niveles de HbA1c, por un período de hasta 3 meses.

EI control metabólico de la diabetes actúa como un elemento clave sobre la modulación de la enfermedad periodontal y de la respuesta reparativa en este tejido.

Figura 2. Conclusiones

\section{CONFLICTOS DE INTERÉS}

Los autores declaran no presentar conflictos de interés de ningún tipo.

\section{BIBLIOGRÁFICAS}

24. Morris HF, Ochi S, Winkler S. Implant survival in patients with type 2 diabetes: Placement to 36 months. Ann Periodontol, 2000; 5: 157-165.

25. Olson JW, Shernoff AF, Tarlow JL, Colwell JA, Scheetz JP, Bingham SF. Dental endosseous implant assessments in a type 2 diabetic population: A prospective study. Int J Oral Maxillofac Implants, 2000; 15: 811-818.

26. Abdulwassie H, Dhanrajani PJ. Diabetes mellitus and dental implants: A clinical study. Implant Dent, 2002; 11: 83-86

27. Moy P, Medina D, Shetty V, Aghaloo T. Dental implant failure rates and associated risk factors. Int J Oral Maxillofac Implants, 2005; 20: 569-577.

28. Oates TW, Dowell S, Robinson M, McMahan CA. Glycemic control and implant stabilization in type 2 diabetes mellitus. J Dent Res, 2009; 88: 367-371.

29. Gooch HL, Hale JE, Fujioka H, Balian G, Hurwitz SR. Alterations of cartilage and collagen expression during fracture healing in experimental diabetes. Connect Tissue Res, 2000; 41: 81-85

30. Amir G, Rosenmann E, Sherman Y, Greenfeld Z, Ne'eman Z, Cohen AM. Osteoporosis in the Cohen diabetic rat: Correlation between histomorphometric changes in bone and microangiopathy. Lab Invest, 2002; 82:1399-1405.

31. Beam HA, Parsons JR, Lin SS. The effects of blood glucose control upon fracture healing in the BB Wistar rat with diabetes mellitus. J Orthop Res, 2002; 20: 1210-1216. 32. Gebauer GP, Lin SS, Beam HA, Vieira P, Parsons JR. Low-intensity pulsed ultrasound increases the fracture callus strength in diabetic BB Wistar rats but does not affect cellular proliferation. J Orthop Res, 2002; 20: 587-592.

33. Lu H, Kraut D, Gerstenfeld LC, Graves DT. Diabetes interferes with the bone formation by affecting the expression of transcription factors that regulate osteoblast differentiation. Endocrinology, 2003; 144: 346-352.

34. Salvi GE, Collins JG, Yalda B, Arnold RR, Lang NP, Offenbacher S. Monocytic TNF alpha secretion patterns in IDDM patients with periodontal diseases. $J$ Clin Periodontol, 1997a; 24: 8-16.

35. Salvi GE, Yalda B, Collins JG, Jones BH, Smith FW, Arnold RR, Offenbacher S. Inflammatory mediator response as a potential risk marker for periodontal diseases in insulin-dependent diabetes mellitus patients. J Periodontol, 1997b; 68: 127-135. 36. Hinz B. Formation and function of the myofibroblast during tissue repair. J Invest Dermatol, 2007; 127: 526-537.

37. Goldberg MT, Han YP, Yan C, Shaw MC, Garner WL. TNF-alpha suppresses alpha-smooth muscle actin expression in human dermal fibroblasts: An implication for abnormal wound healing. J Invest Dermatol, 2007; 127: 2645-2655.

38. Murillo J, Wang Y, Xu X, Klebe RJ, Chen Z, Zardeneta G, Pal S, Mikhailova $M$, Steffensen B. Advanced glycation of type I collagen and fibronectin modifies periodontal cell behavior. J Periodontol, 2008; 79: 2190-2199.

39. Chong SAC, Lee W, Arora PD, Laschinger C, Young EWK, Simmons CA, Manolson M, Sodek J, McCulloch CA. Methylglyoxal inhibits the binding step of collagen phagocytosis. J Biol Chem, 2007; 282: 8510-8520.

40. Grossi SG, Skrepcinski FB, DeCaro T, Robertson DC, Ho AW, Dunford RG et al. Treatment of periodontal disease in diabetics reduces glycated hemoglobin. $J$ Periodontol, 1997; 68: 713-719.

41. Janket SJ, Wightman A, Baird AE, Van Dyke TE, Jones JA. Does periodontal treatment improve glycemic control in diabetics patients? A meta-analysis of interventional studies. J Dent Res, 2005; 84: 1154-1159.

42. Vergnes JN. Treating periodontal disease may improve metabolic control in diabetics. Evid Based Dent, 2010; 11: 73-74.

43. Simpson TC, Needleman I, Wild SH, Moles DR, Mills EJ. Treatment of periodontal disease for glycaemic control in people with diabetes. Cochrane Database Syst Rev, 2010 May 12; (5): CD004714.

44. Auyeung L, Wang PW, Lin RT, Hsieh CJ, Lee PY, Zhuang RY, Chang HW. Evaluation of periodontal status and effectiveness of non-surgical treatment in patients with type 2 diabetes mellitus in Taiwan for a one-year period. J Periodontol, 2011 Jun 21. [Epub ahead of print]. 